\title{
Enhanced expression of KIF4A in osteosarcoma predicts a poor prognosis and facilitates tumor growth by activation of the MAPK pathway
}

\author{
DONGSHENG ZHU* ${ }^{*}$ XIANGFEI XU* ${ }^{*}$ MING ZHANG* ${ }^{*}$ and TONG WANG \\ Department of Pediatric Orthopedic Surgery, Lianyungang No. 1 People's Hospital \\ Affiliated to Xuzhou Medical University, Lianyungang, Jiangsu 222000, P.R. China
}

Received May 5, 2021; Accepted August 13, 2021

DOI: $10.3892 /$ etm.2021.10774

\begin{abstract}
The present study aimed to explore the prognostic value and role of kinesin family member 4A (KIF4A) expression in human osteosarcoma. KIF4A expression was evaluated in human osteosarcoma tissues from The Cancer Genome Atlas and Gene Expression Omnibus datasets. Reverse transcription-quantitative PCR was then applied to assess KIF4A level in both osteosarcoma cell lines and tissues. The association between KIF4A expression and clinical results in patients with osteosarcoma was detected by survival analysis. MTT assays and colony formation assays were used to evaluate the effects of KIF4A on osteosarcoma cell proliferation. The results indicated that the level of KIF4A was increased and associated with a poor prognosis in osteosarcoma tissues. Knockdown of KIF4A was shown to inhibit osteosarcoma cellular proliferation by affecting the MAPK pathway. The level of KIF4A was high in the human osteosarcoma tissues and this could be considered as a tumor induction gene, which may be used as an indicator of prognosis.
\end{abstract}

\section{Introduction}

Globally, the most common primary malignant bone tumor among teenagers and young adults is osteosarcoma (1). Due to surgery being combined with chemotherapy, the 5-year survival rate for patients with osteosarcoma has reached $50-80 \%$ (2). However, $\sim 80 \%$ of osteosarcoma patients will develop local recurrence or metastasis after surgical

Correspondence to: Dr Dongsheng Zhu or Dr Tong Wang, Department of Pediatric Orthopedic Surgery, Lianyungang No. 1 People's Hospital Affiliated to Xuzhou Medical University, 182 Tongguan North Road, Lianyungang, Jiangsu 222000, P.R. China E-mail: zhudongsheng@tmu.edu.cn

E-mail: 411524886@qq.com

"Contributed equally

Key words: kinesin family member 4A, pediatrics, proliferation, osteosarcoma, MAPK pathway treatment (3). Therefore, in order to further improve the survival rate of patients with osteosarcoma, there is an urgent requirement to develop new diagnostic biomarkers and innovative treatment strategies. Prior studies have suggested that there are a wide range of genetic and molecular alterations in osteosarcoma $(4,5)$; however, the highly complex molecular mechanism of osteosarcoma has not been elucidated.

Kinesin superfamily proteins are responsible for the movement of membrane-bound compartments and transport vesicles (6). This family contains 45 genes, known as kinesins or kifs, which are divided into 14 different families (7). Kifs play a vital role in mitosis, especially during spindle formation and cytokinesis (8). Kinesin family member 4A (KIF4A) is one member that appears to play a role in the regulation of gene expression and heterochromatin formation (9). A number of studies have shown that abnormal expression of KIF4A can lead to tumorigenesis, including that of breast, lung, colorectal and liver cancer (10-13). However, the role of KIF4A in osteosarcoma is unclear. In the present study, the aim was to explore the role of KIF4A in human osteosarcoma and confirm whether it could be considered as a tumor induction gene, which may be used an indicator of prognosis.

\section{Materials and methods}

Bioinformatics analyses. KIF4A expression level and Kaplan-Meier survival analysis were obtained using the Gene Expression Profiling Interactive Analysis (GEPIA) website (http://gepia.cancer-pku.cn/index.html) using the term 'KIF4A' in the 'Gene' field and 'SARC' in the 'Datasets Selection' field. Differential expression of KIF4A was defined with the following significance cutoff levels: $\mid \log _{2} \mathrm{FCl}>1$ and $\mathrm{P}<0.01$. For the Kaplan-Meier survival analysis, the same terms were used and the group cutoff for KIF4A was the median expression value. The Gene Expression Omnibus (GEO) database (www.ncbi.nlm. gov/geo) was used to download the GSE28424 dataset (14). After using 'osteosarcoma' as the search term, the GSE28424 dataset was selected, as the study gives a comprehensive analysis of osteosarcoma mRNA and provides new insight into the complex genetic mechanisms of tumor development and the progression of osteosarcoma. The common differentially expressed genes were obtained using the GEO2R online tool (http://www.ncbi. 
nlm.nih.gov/geo/geo2r/). $\mathrm{P}<0.05$ and $\mid \log _{2} \mathrm{FCl}>1$ were set as cut-off criteria and KIF4A was selected.

Patients. A total of 72 paired primary osteosarcoma and adjacent noncancerous tissues were retrospectively obtained from patients who received palliative surgery or radical resection at Lianyungang No. 1 People's Hospital Affiliated to Xuzhou Medical University (Lianyungang, China) between January 2005 and December 2018 (age range, 2-14 years; average age, $9.47 \pm 3.30$ years). None of the enrolled patients received radiotherapy or chemotherapy before surgery. All patients underwent chemotherapy after surgery. Inclusion criteria: Osteosarcoma patients without radiotherapy or chemotherapy before surgery. Exclusion criteria: i) Osteosarcoma patients received radiotherapy or chemotherapy before surgery; ii) patients refused to sign the informed consent form. The histological diagnosis of osteosarcoma was confirmed by two professional pathologists and conformed to the World Health Organization's and the American Joint Committee on Cancer histological criteria (15). The collected specimens were snap frozen in liquid nitrogen and stored at $-80^{\circ} \mathrm{C}$ promptly until RNA extraction. All enrolled patient guardians provided written informed consent. This research conformed to the principles of the Declaration of Helsinki and was approved by the Ethics Committee of The Lianyungang No. 1 People's Hospital Affiliated to Xuzhou Medical University.

Cell lines. The human normal osteoblastic hFOB cell line and the human osteosarcoma MG63, U2OS, HOS and Saos2 cell lines were purchased from the American Type Culture Collection and cultured in DMEM (Invitrogen; Thermo Fisher Scientific, Inc.) supplemented with $10 \%$ fetal bovine serum (Gibco; Thermo Fisher Scientific, Inc.) in a humidified incubator containing $5 \% \mathrm{CO}_{2}$ at $37^{\circ} \mathrm{C}$.

$R N A$ isolation and reverse transcription-quantitative PCR $(R T-q P C R)$. TRIzol ${ }^{\circledR}$ reagent (Invitrogen; Thermo Fisher Scientific, Inc.) was used to extract RNA from cell lines (hFOB, MG63, U2OS, HOS and Saos2) and tissues (primary osteosarcoma and adjacent noncancerous tissues). Following RNA extraction, the EasyScript One Step gDNA Removal and cDNA Synthesis SuperMix (TransGen Biotech Co., Ltd.) was used to acquire the first strand cDNA according to the manufacturer's instructions. Next, RT-qPCR was performed using SYBR-Green Master Mix kit (Roche Diagnostics) with an Applied Biosystems 7900 Real-Time PCR System (Thermo Fisher Scientific, Inc.). The reaction conditions were as follows: Pre-denaturation at $95^{\circ} \mathrm{C}$ for $15 \mathrm{~min}$; followed by 35 cycles of denaturation at $95^{\circ} \mathrm{C}$ for $15 \mathrm{sec}$, annealing at $58^{\circ} \mathrm{C}$ for $30 \mathrm{sec}$ and extension at $72^{\circ} \mathrm{C}$ for $5 \mathrm{~min}$; and final extension at $72^{\circ} \mathrm{C}$ for 15 min. Expression levels of RNA were calculated based on the comparative $2^{-\Delta \Delta C q}$ method. RT-qPCR was performed as in previous publications $(16,17)$. The level of $\beta$-actin was used to normalize the relative LIF4A expression level. Experiments were performed with the following primers: KIF4A forward, 5'-TGGTGTGGAAACAAGCAGTGTG-3' and reverse, 5'-CAC CCTGTTGTTGCTGTAGCCAAA-3'; and $\beta$-actin forward, 5'-TCACCCACACTGTGCCCATCTACGA-3' and reverse, 5'-CAGCGGAACCGCTCATTGCCAATGG-3'. All experiments were performed in triplicate.
siRNA transfection. siRNA oligos for KIF4A, commercially constructed by Shanghai GenePharma Co., Ltd., were used to perform knockdown experiments. KIF4A-siRNA oligo sequences were as follows: Sense, 5'-GGAAUGAGGUUGUGA UCUUTT-3' and anti-sense, 5'-AAGAUCACAACCUCA UUCCTT-3'. Non-silencing siRNA (sense, 5'-UUCUCCGAAC GUGUCACGUTT-3' and antisense, 5'-ACGUGACACGUUC GGAGAATT-3') was used as a negative control. Before transfecting siRNA (20 nM) with Lipofectamine ${ }^{\circledR} 3000$ (Invitrogen; Thermo Fisher Scientific, Inc.), the cells were cultured in growth media in a 6 -well plate until $70 \%$ confluence was reached according to the manufacturer's instructions. Transfection was performed at $37^{\circ} \mathrm{C}$ for $6 \mathrm{~h}$ before changing the transfection medium with full culture medium. Then cells were harvested $48 \mathrm{~h}$ after transfection, and then western blotting or RT-qPCR analyses were performed.

Cell proliferation assay. At $48 \mathrm{~h}$ post-siRNA transfection, transfected cells at a density of 1,000 cells per well were seeded in 96-well plates, with 5 replicate wells. A total of $10 \mu \mathrm{l}$ of $5 \mathrm{mg} / \mathrm{ml}$ MTT was added into each well at $0,24,48$ and $72 \mathrm{~h}$ after transfection, and then the cells were cultured for $2 \mathrm{~h}$ in an incubator at $37^{\circ} \mathrm{C}$. Then, $150 \mu \mathrm{l}$ DMSO was added per well. Finally, a microplate reader was used to measure the absorbance at $490 \mathrm{~nm}$. All viability experiments were performed in triplicate.

Colony formation assay. At $6 \mathrm{~h}$ post-transfection, MG63 and U2OS cells were resuspended as a single-cell suspension using trypsin, and then seeded into 6-well plates with 2,000 cells/well, with $2 \mathrm{ml}$ complete medium added to each well. Next, the 6-well plates were placed in an incubator at $37^{\circ} \mathrm{C}$. After 14 days, $4 \%$ paraformaldehyde was used to fix the cells for $20 \mathrm{~min}$ at room temperature, $0.1 \%$ crystal violet was applied to stain the cells for $30 \mathrm{~min}$ at room temperature, and then tap water was used to rinse the cells, which were photographed for manual counting. Colonies consisted of $>50$ cells.

Western blot analysis. After using pre-iced PBS to wash the cells, the cells were lysed with RIPA buffer for $30 \mathrm{~min}$, and then centrifuged to collect the supernatant at $14,000 \mathrm{x} g$ for $15 \mathrm{~min}$ at $4^{\circ} \mathrm{C}$. Bradford protein assay (Bio-Rad Laboratories, Inc.) was used to conduct protein quantitation; then per lane was loaded $30 \mu \mathrm{g}$ of protein. Electrophoresis was performed with $10 \%$ SDS-PAGE, and the separated proteins were transferred onto nitrocellulose membranes. After transferring the protein to membranes, $5 \%$ skimmed milk was used to block the membranes for $1 \mathrm{~h}$ at room temperature, followed by incubation with the following primary antibodies at $4^{\circ} \mathrm{C}$ overnight: Rabbit anti-KIF4A (1:1,000 dilution; catalog no. ab124903), anti-GAPDH (1:2,500 dilution; catalog no. ab9485), anti-ERK (1:5,000 dilution; ab265600), anti-MEK (1:20,000 dilution; catalog no. ab178876); anti-cAMP responsive element binding protein (CREB; 1:1,000 dilution; catalog no. ab31387) (all Abcam). Subsequently, HRP-conjugated secondary antibodies (1:5,000 dilution; catalog no. S0001; Affinity Biosciences) were added for $1 \mathrm{~h}$ at room temperature. Pierce ${ }^{\mathrm{TM}}$ ECL Western Blotting Substrate (cat. no. 32109; Thermo Fisher Scientific, Inc.) was used to visualize the bound antibodies. The levels of protein were evaluated using chemiluminescence detection 

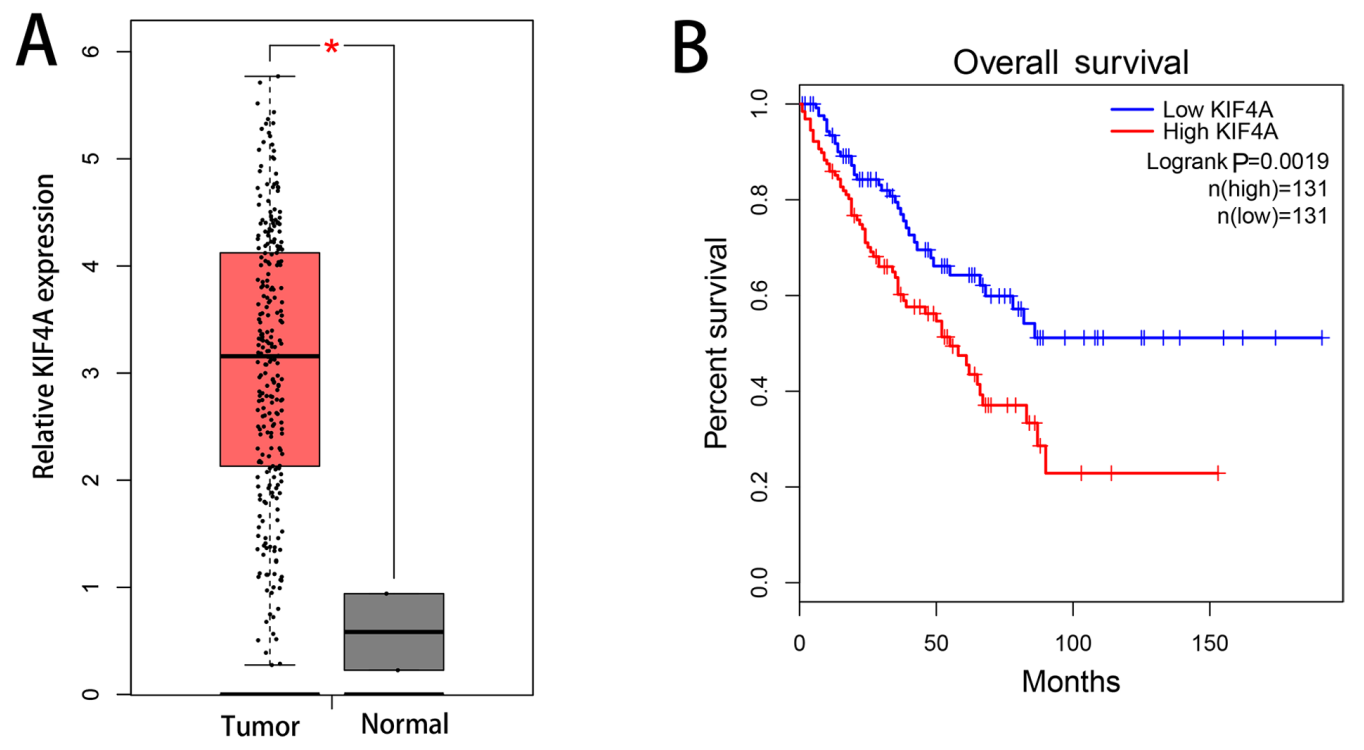

Figure 1. Expression level of KIF4A in osteosarcoma tissues and normal tissues (The Cancer Genome Atlas database), and the associated survival analysis. (A) KIF4A expression was significantly higher in osteosarcoma tissues compared with that in normal tissues. (B) Overall survival with regard to patients with high and low KIF4A expression. ${ }^{*} \mathrm{P}<0.05$. KIF4A, kinesin family member 4A.

system (GE Healthcare Biosciences), and image analysis was performed using ImageJ software (version 1.42; National Institutes of Health). Western blotting was performed as in a previous publication (17).

Statistical analysis. Data are presented as the mean \pm standard deviation. Paired or unpaired Student's t-test or the Mann-Whitney U test was used to compare the continuous variables in two groups. One-way ANOVA was adopted to compare the continuous variables among multiple groups, followed by Dunnett's post hoc test. The differences in categorical variables were analyzed with Pearson's $\chi^{2}$ and Fisher's exact tests and data were presented as $\mathrm{n}(\%)$. The prognostic value of patient survival was estimated by Kaplan-Meier method and log-rank tests. Receiver operating curve (ROC) analysis was used to assess predictive value of biomarkers, together with calculation of the area under the ROC curve (AUC), and 'sensitivity + specificity -1' was used as the best cut-off value. Pearson correlation was used to evaluate the strength of linear correlation between two continuous variables. GraphPad Prism 8.0 (GraphPad Software, Inc.) was used to analyze all statistical data. $\mathrm{P}<0.05$ was used to indicate a statistically significant difference, and all P-values were two-sided.

\section{Results}

KIF4A upregulation in osteosarcoma. Using analysis of a clinical patient database, by GEPIA, the expression level of KIF4A in sarcoma tissues $(n=262)$ was found to be upregulated in comparison with that non-sarcoma tissues $(n=2)$ at the mRNA level (Fig. 1A). Upregulated expression of KIF4A was also associated with poor overall survival (Fig. 1B).

Analysis of the GEO dataset GSE28424 indicated that KIF4A expression was upregulated in osteosarcoma $(n=19)$ compared with that in non-osteosarcoma $(n=4)$ (Fig. 2A and B). The sensitivity and specificity of KIF4A was evaluated by ROC-AUC, and the result showed that the AUC was 0.83, suggesting that KIF4A should be considered as a potential biomarker of osteosarcoma cases (Fig. 2C).

Compared with that in the normal osteoblastic hFOB cell line, the level of KIF4A in the osteosarcoma MG63, U2OS, HOS and Saos2 cell lines was higher according to RT-qPCR analysis (Fig. 3A). In the 72 pairs of human osteosarcoma and adjacent tissue collected, there were similar results (Fig. 3B).

KIF4A expression level is associated with a poor outcome in patients with osteosarcoma. Using the paired patient tissues, the AUC of KIF4A was determined to be 0.87 (Fig. 3C). ROC analysis was used to acquire the best cut-off value and then two groups were obtained, the low KIF4A expression group $(n=42)$ and the high KIF4A expression group $(n=30)$ were created (Table I). Clinical stage (15), distant metastasis and response to chemotherapy were found to be significantly associated with high KIF4A expression $(\mathrm{P}=0.018, \mathrm{P}=0.042$ and $\mathrm{P}=0.020$, respectively). However, anatomical location, sex, age, lactate dehydrogenase level, alkaline phosphatase level, and tumor size were not associated with the expression of KIF4A. Moreover, it was found that high expression level of KIF4A was associated with poor overall survival in patients with osteosarcoma $(\mathrm{P}=0.031$; Fig. 3D).

Silencing of KIF4A inhibits the proliferation of osteosarcoma cells. KIF4A siRNA plasmids were used to knock down KIF4A expression in the osteosarcoma U2OS and MG63 cell lines, which were selected as they are derived from children and teenagers. Next, RT-qPCR was performed to show the efficiency of the KIF4A knockdown (Fig. 4A and B). The transfection and expression efficiency was also confirmed by western blotting (Fig. 4C). MTT assays were performed to assess proliferation in KIF4A-siRNA-transfected osteosarcoma cells. Results indicated that KIF4A knockdown inhibited U2OS and MG63 cell proliferation (Fig. 4D and E). Similarly, the colony formation assays found that KIF4A knockdown 
Table I. Association between KIF4A level and the clinicopathological features of osteosarcoma.

\begin{tabular}{|c|c|c|c|c|}
\hline \multirow[b]{2}{*}{ Clinicopathological features } & \multirow[b]{2}{*}{ Number of cases } & \multicolumn{2}{|c|}{ KIF4A expression } & \multirow[b]{2}{*}{ P-value } \\
\hline & & High, n (\%) & Low, n (\%) & \\
\hline Age, years & & & & 0.417 \\
\hline$<8$ & 19 & $6(31.58)$ & $13(68.42)$ & \\
\hline$\geq 8$ & 53 & $24(45.28)$ & $29(54.72)$ & \\
\hline Sex & & & & 0.809 \\
\hline Male & 41 & $18(43.90)$ & $23(56.10)$ & \\
\hline Female & 31 & $12(38.71)$ & $19(61.29)$ & \\
\hline Tumor size, $\mathrm{cm}$ & & & & 0.634 \\
\hline$<8$ & 33 & $15(45.45)$ & $18(54.55)$ & \\
\hline$\geq 8$ & 39 & $15(38.46)$ & $24(61.54)$ & \\
\hline Anatomical location & & & & 0.063 \\
\hline Tibia/femur & 60 & $28(46.67)$ & $32(53.33)$ & \\
\hline Elsewhere & 12 & $2(16.67)$ & $10(83.33)$ & \\
\hline Serum level of lactate dehydrogenase & & & & 0.305 \\
\hline Elevated & 49 & $18(36.73)$ & $31(63.27)$ & \\
\hline Normal & 23 & $12(52.17)$ & $11(47.83)$ & \\
\hline Serum level of alkaline phosphatase & & & & 0.116 \\
\hline Elevated & 51 & $18(35.29)$ & $33(64.71)$ & \\
\hline Normal & 21 & $12(57.14)$ & $9(42.86)$ & \\
\hline Clinical stage & & & & 0.018 \\
\hline I & 23 & $5(21.74)$ & $18(78.26)$ & \\
\hline II & 35 & $15(42.86)$ & $20(57.14)$ & \\
\hline III & 14 & $10(71.43)$ & $4(28.57)$ & \\
\hline Distant metastasis & & & & 0.042 \\
\hline Absent & 61 & $22(36.07)$ & $39(63.93)$ & \\
\hline Present & 11 & $8(72.73)$ & $3(27.27)$ & \\
\hline Response to chemotherapy & & & & 0.020 \\
\hline Good & 56 & $19(33.93)$ & $37(66.07)$ & \\
\hline Poor & 16 & $11(68.75)$ & $5(31.25)$ & \\
\hline
\end{tabular}

KIF4A, kinesin family member 4A.

inhibited colony formation (Fig. 4F). Taken together, both MTT and colony formation assay findings showed that osteosarcoma cell proliferation was inhibited when KIF4A was knocked down.

KIF4A knockdown may suppress MAPK signaling pathway activity in osteosarcoma cells. The MAPK signaling pathway is an important regulatory pathway that regulates the growth of cancer cells. Positive correlations were found between KIF4A and the five key genes in the MAPK signaling pathway, as determined in GEPIA using bioinformatics analysis of co-expression (Fig. 5A-F). To confirm our hypothesis of a positive correlation between the KIF4A and MAPK signaling pathways, the protein expression levels of ERK, MEK and CREB were obtained after knockdown of KIF4A in osteosarcoma U2OS and MG63 cells. The expression of the three proteins was found to be significantly decreased when KIF4A was knocked down (Fig. 6). These findings show that KIF4A may facilitate tumor proliferation by affecting the MAPK pathway.

\section{Discussion}

In the pediatric age group, osteosarcoma is the most frequent malignant tumor of the bone, with a poor prognosis (4). Currently, the therapeutic efficacy of osteosarcoma treatment strategy is not ideal, and it is especially important to find a sensitive biomarker for the diagnosis of early phase osteosarcoma and as a target for treatment. Osteosarcoma is a disease resulting from a combination of multiple genes (5). However, the key genes in osteosarcoma have not been determined. Hence, it is still necessary to explore novel therapeutic targets.

The results of the present study suggested that the expression level of KIF4A was high in osteosarcoma, as observed in other malignant tumors, as reported by previous studies $(11,18-21)$. In osteosarcoma, KIF4A upregulation has been confirmed by 
A

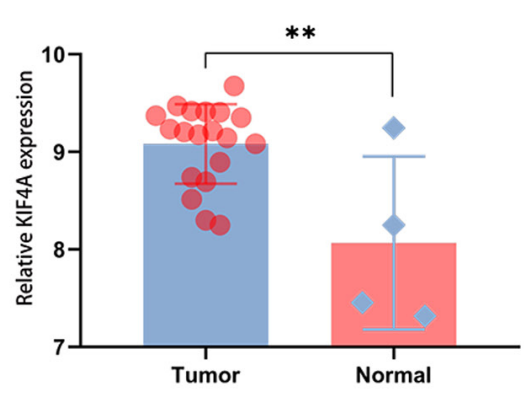

C

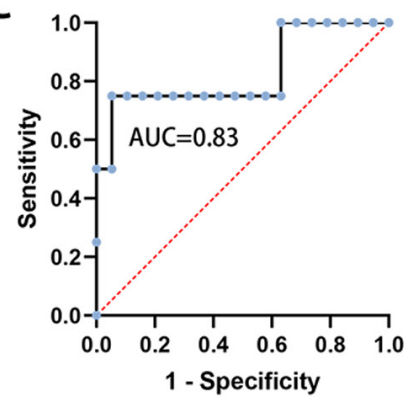

B

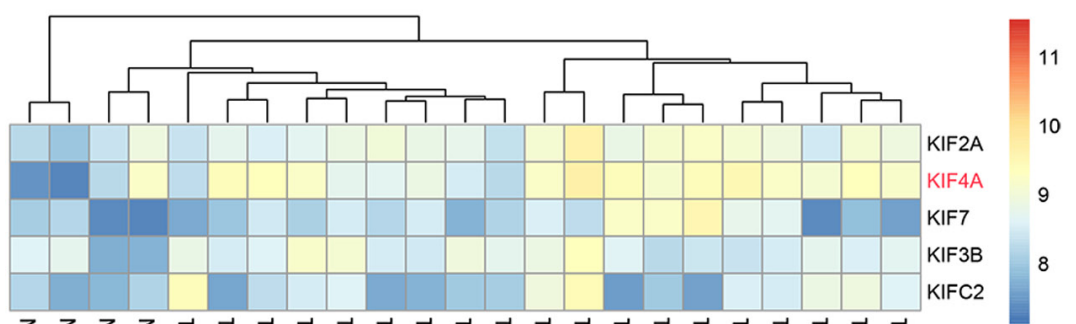

Figure 2. KIF4A is upregulated in osteosarcoma tissues (Gene Expression Omnibus dataset GSE28424). (A and B) KIF4A level was higher in tumor samples than in normal samples. (C) Receiver operating characteristic curve showing an AUC value of 0.83 , indicating that the high sensitivity and specificity of KIF4A expression for the diagnosis of osteosarcoma. ${ }^{* *} \mathrm{P}<0.01$. KIF4A, kinesin family member $4 \mathrm{~A}$.
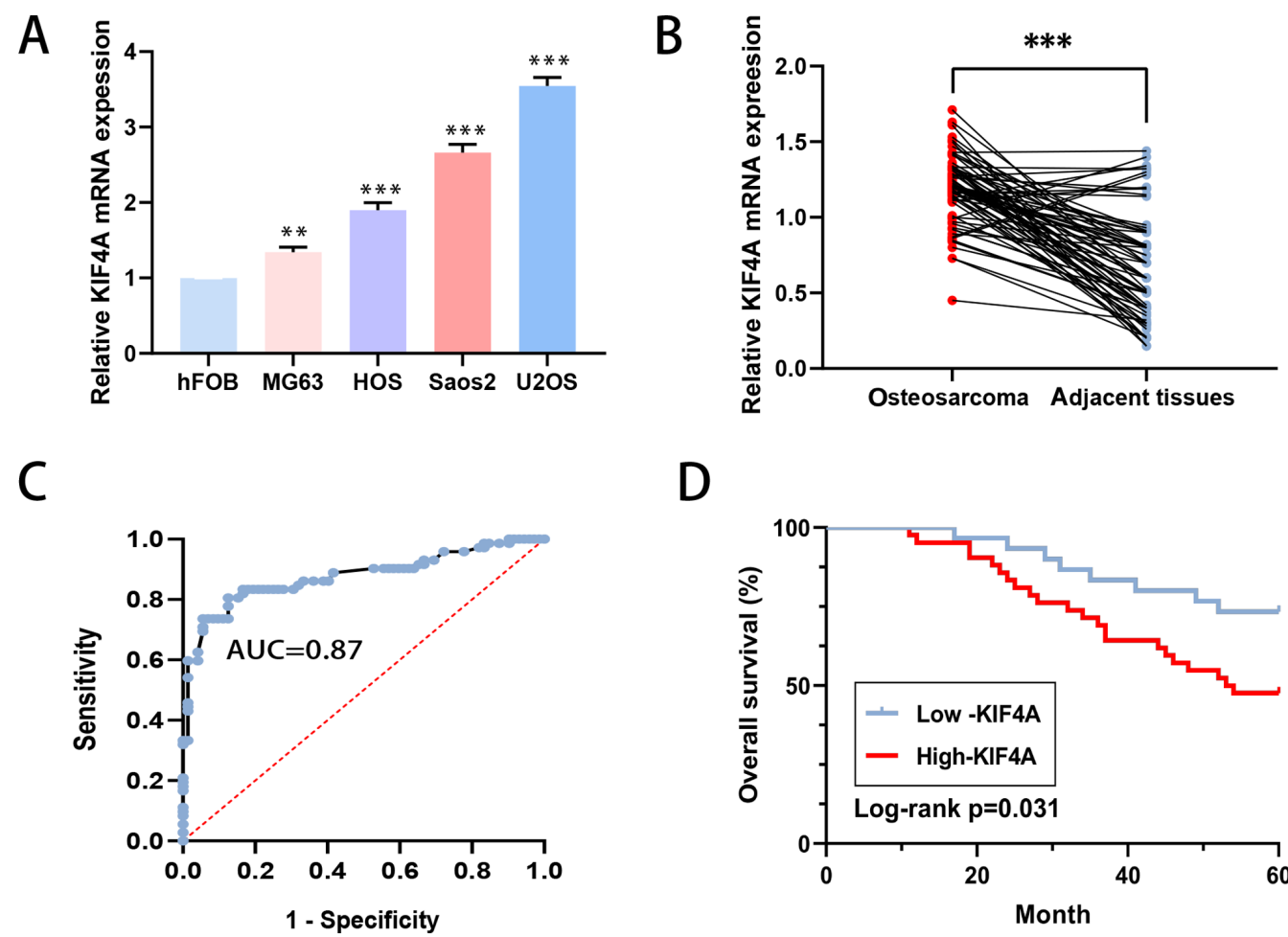

D

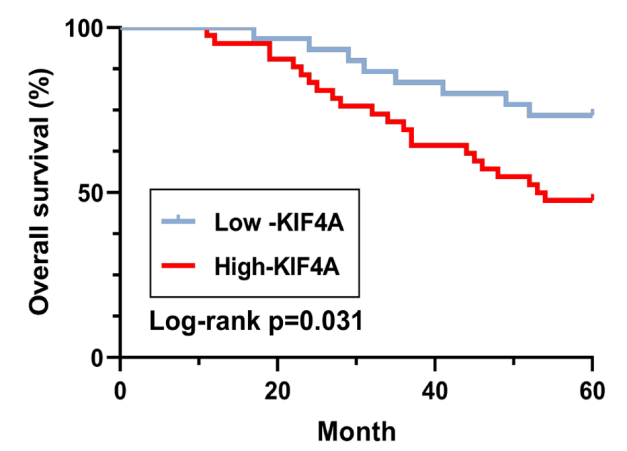

Figure 3. KIF4A is upregulated in osteosarcoma tissues and associated with a poor prognosis. (A) Osteosarcoma cell lines (MG63, HOS, Saos2 and U2OS) had a higher KIF4A level than hFOB cells. (B) Osteosarcoma tissues had a higher KIF4A level than adjacent patient tissues. (C) Receiver operating characteristic curve of KIF4A for osteosarcoma tissues. (D) Survival curves for patients with osteosarcoma. ${ }^{* *} \mathrm{P}<0.01$ and ${ }^{* * * *} \mathrm{P}<0.001$. KIF4A, kinesin family member $4 \mathrm{~A}$.

bioinformatics analysis, and this has been validated by cell experiments (22). However, to the best of our knowledge, no clinical data exists. The present study also found KIF4A upregulation in osteosarcoma. Furthermore, KIF4A was found to promote the proliferation of osteosarcoma cells through the MAPK signaling pathway. In the present study, in the ROC curve analysis based on the patient tissue data, the AUC was 0.87 , meaning that KIF4A was a highly sensitive diagnostic predictor of osteosarcoma. Furthermore, in the patients with osteosarcoma, a higher expression level of KIF4A was associated with a shorter overall survival 
A

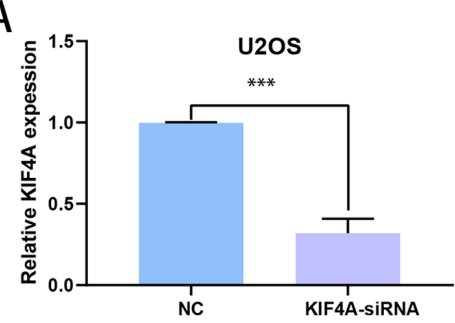

B

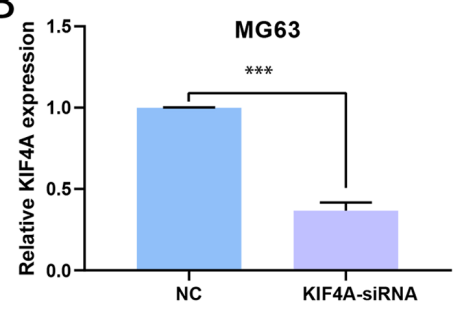

C

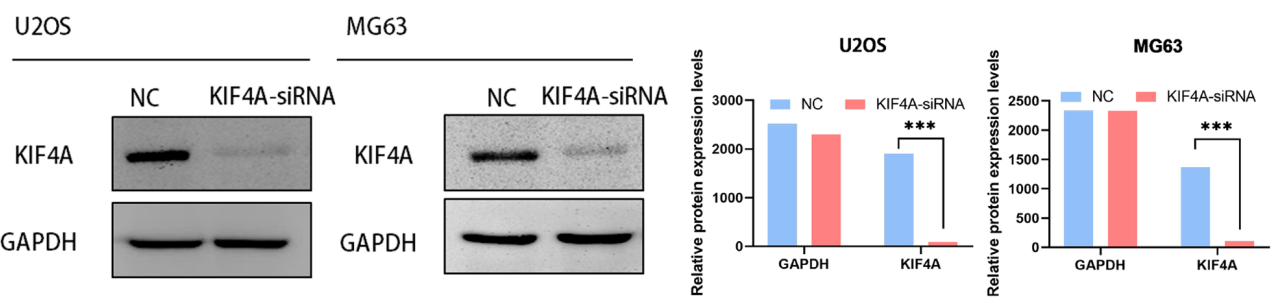

D

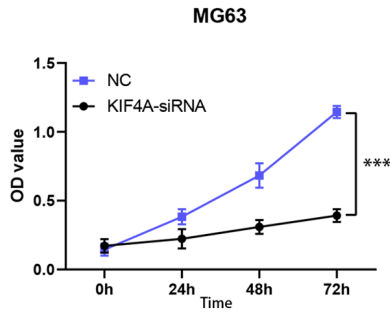

E

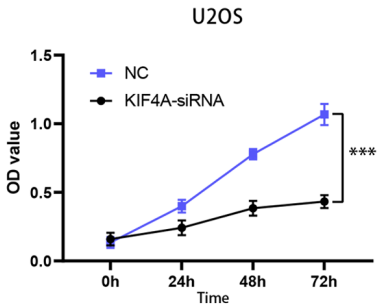

F

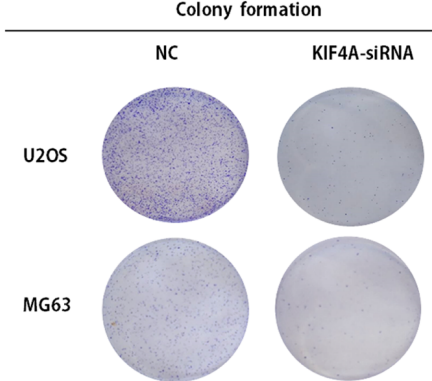

Figure 4. Proliferation of osteosarcoma cells is inhibited after knockdown of KIF4A. (A) Transfection efficiency of si-KIF4A in the U2OS cell line by RT-qPCR analysis. (B) Transfection efficiency of si-KIF4A in the MG63 cell line by RT-qPCR analysis. (C) Transfection efficiency of si-KIF4A in osteosarcoma U2OS and MG63 cell lines by western blotting analysis. (D) Proliferation in the U2OS cell line after KIF4A knockdown according to MTT analysis. (E) Proliferation in the MG63 cell line after KIF4A knockdown according to MTT analysis. (F) Changes in colony formation ability in the U2OS and MG63 cell lines after KIF4A knockdown. ${ }^{* * *} \mathrm{P}<0.001$. KIF4A, kinesin family member 4A; RT-qPCR, reverse transcription-quantitative polymerase chain reaction; si, small interfering; OD, optical density.
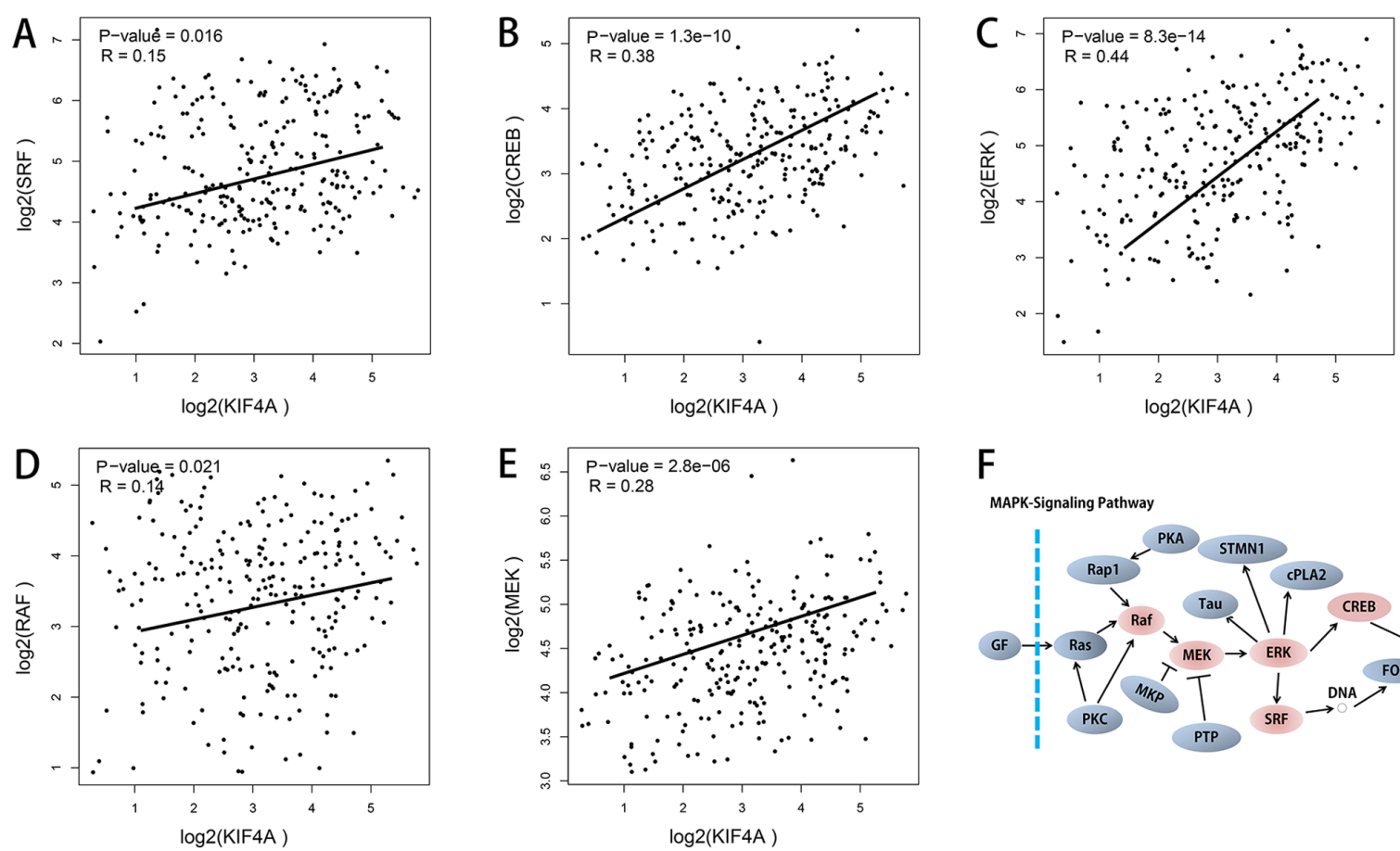

$\mathrm{F}$ MAPK-Signaling Pathway

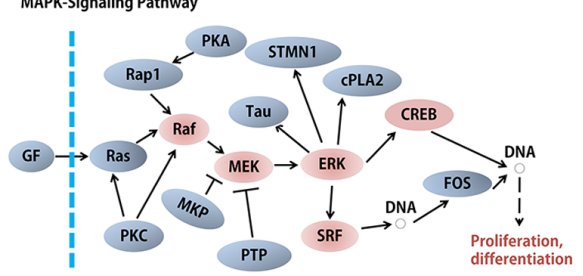

Figure 5. KIF4A is positively correlated with the MAPK signaling pathway in osteosarcoma (Gene Expression Profiling Interactive Analysis database). The correlation of KIF4A expression with (A) SRF, (B) CREB, (C) ERK, (D) RAF and (E) MEK by Pearson's correlation analysis in osteosarcoma. (F) The MAPK signaling pathway. These correlations were only observed in osteosarcoma samples. KIF4A, kinesin family member 4A; SRF, serum response factor; CREB, cAMP-responsive element binding protein; ERK, mitogen-activated protein kinase 3; RAF, zinc fingers and homeoboxes 2; MEK, mitogen-activated protein kinase kinase 7. 

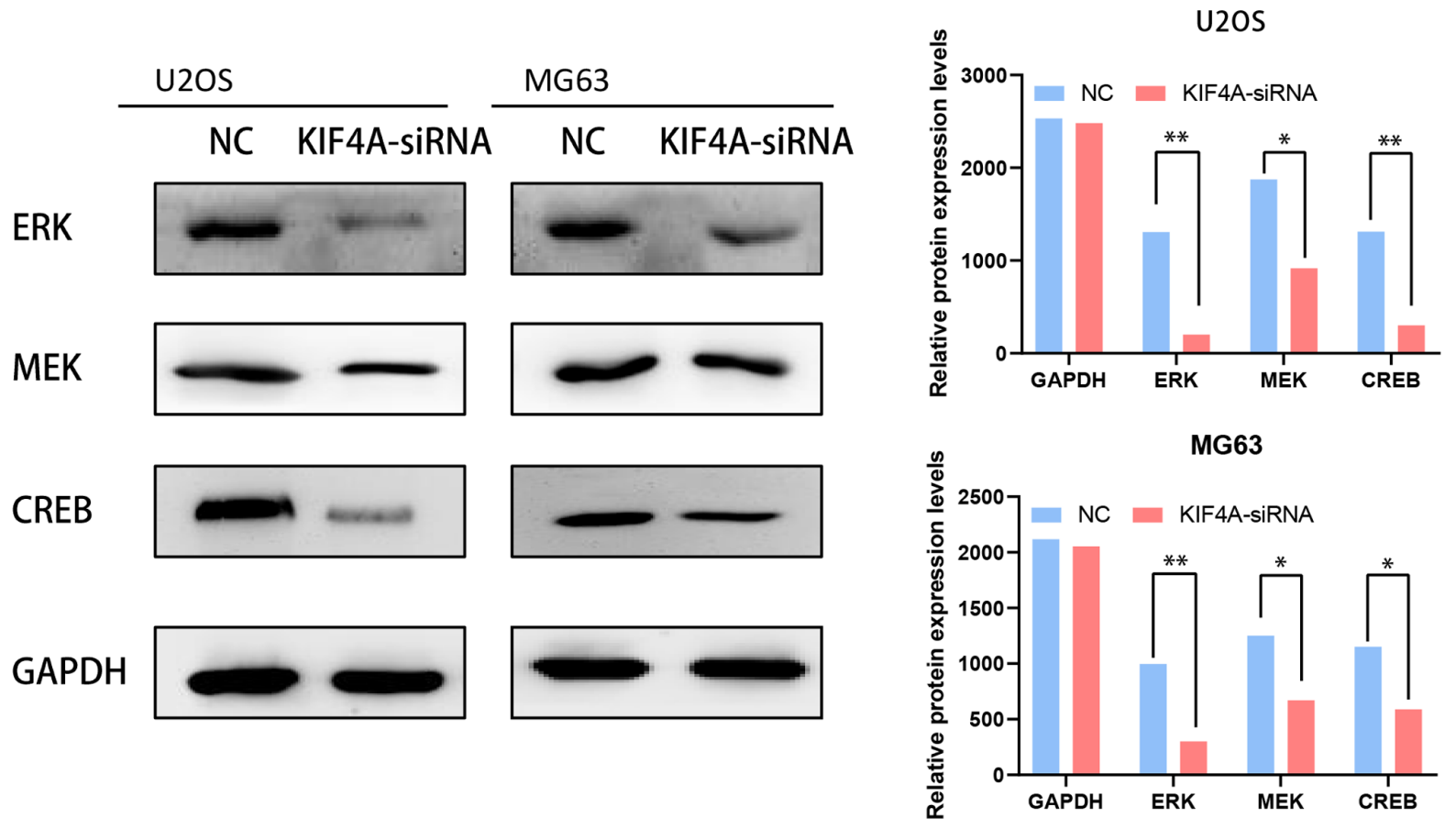

Figure 6. Knockdown of KIF4A inhibits the MAPK signaling pathway. ${ }^{*} \mathrm{P}<0.05,{ }^{* *} \mathrm{P}<0.01$. KIF4A, kinesin family member 4A; CREB, cAMP responsive element binding protein; si, small interfering.

time, according to Kaplan-Meier analysis $(\mathrm{P}=0.031)$. KIF4A may therefore be considered as a therapeutic target in osteosarcoma and a factor that affects the outcome of patients with this disease.

KIF4A is a member of the kif subfamily; various studies have previously suggested that KIF4A has a critical role in tumorigenesis and tumor progression $(11,20)$. KIF4A, in humans, is a motor protein for the action of microtubules and plays a role in spindle formation, as well as in the regulation of chromosome segregation (23). In 2007, scientists first discovered that KIF4A was upregulated in carcinoma of the lungs and that silencing KIF4A using siRNA can suppress the invasive activity of tumor cells (11). In a recent study of renal cancer, Liu et al (24) reported that KIF4A might be an independent factor that predicts recurrence-free survival and overall survival in renal carcinoma. These findings are consistent with the experimental results from the present study.

The present study also found that the proliferation of osteosarcoma cells was suppressed when KIF4A expression was inhibited. In colorectal cancer, KIF4A can mediate p21 to arrest the cell cycle, promoting the proliferation of tumor cells. The protein level of ERK, MEK and AKT were decreased when silencing KIF4A, indicating that the PI3K/AKT signaling pathway was inactivated (12). However, in the present study, when KIF4A activation was inhibited, the expression of AKT was not changed. KIF4A downregulation resulted in changes in the protein expression levels of MEK, ERK and CREB. Further research is required for this different mechanism.

As reported in a number of studies, upregulation of KIF4A is associated with a number of different cancer types $(12,19,22)$, but the molecular mechanisms underlying KIF4A function in osteosarcoma are not well understood. The present study revealed the association between KIF4A and clinicopathological features in osteosarcoma, and found that
KIF4A indeed promoted the proliferation of osteosarcoma via the MAPK signaling pathway.

Overall, KIF4A in osteosarcoma may be considered as a potential target for tumor therapy. This finding should help to normalize potential new targets for the diagnosis and treatment of osteosarcoma. However, there were some limitations faced in the present study. First, it was only a retrospective study; second, the study was conducted in vitro; third, the clinical sample size was not large at only 72 cases; fourth, the GEPIA data only contained 2 normal samples, and the GEO data contained 4, making any statistical analysis difficult to interpret. We acknowledge the limited number of control samples as a limitation in this study. Fifth, only the proliferation of osteosarcoma cells affected by KIF4A was analyzed. Finally, only knockdown experiments, but no overexpression experiments, were performed. Future studies will continue to reveal the role of KIF4A in osteosarcoma.

In conclusion, in the present study, KIF4A was found to promote tumor progression via activation of the MAPK signaling pathway in osteosarcoma, and could be considered as a novel biomarker and potential target for osteosarcoma treatment.

\section{Acknowledgements}

Not applicable.

\section{Funding}

No funding was received.

\section{Availability of data and materials}

The datasets used and/or analyzed during the current study are available from the corresponding author on reasonable request. 


\section{Authors' contributions}

DZ and TW conceived and directed the project. DZ contributed to the writing of the manuscript. DZ, XX and MZ analyzed the data. DZ, XX and MZ collected the clinical data. DZ, XX, MZ and TW confirmed the authenticity of the raw data. All authors have read and approved the final manuscript.

\section{Ethics approval and consent to participate}

All of the enrolled patient guardians provided written informed consent. This research conformed to the principles of the Declaration of Helsinki and was approved by the Ethics Committee of The Lianyungang No. 1 People's Hospital Affiliated to Xuzhou Medical University (December 2018; approval no. 20180104; Lianyungang, China).

\section{Patient consent for publication}

Not applicable.

\section{Competing interests}

The authors declare that they have no competing interests.

\section{References}

1. Ritter J and Bielack SS: Osteosarcoma. Ann Oncol 21 (Suppl 7): vii320-vii325, 2010.

2. Mirabello L, Troisi RJ and Savage SA: Osteosarcoma incidence and survival rates from 1973 to 2004: Data from the Surveillance, Epidemiology, and End Results Program. Cancer 115: 1531-1543, 2009.

3. Marina N, Gebhardt M, Teot L and Gorlick R: Biology and therapeutic advances for pediatric osteosarcoma. Oncologist 9: 422-441, 2004

4. Joko R, Yamada D, Nakamura M, Yoshida A, Takihira S, Takao T, Lu M, Sato K, Ito T, Kunisada T, et al: PRRX1 promotes malignant properties in human osteosarcoma. Transl Oncol 14: 100960, 2021.

5. Tian W, Li Y, Zhang J, Li J and Gao J: Combined analysis of DNA methylation and gene expression profiles of osteosarcoma identified several prognosis signatures. Gene 650: 7-14, 2018.

6. Hirokawa $\mathrm{N}$ and Tanaka Y: Kinesin superfamily proteins (KIFs): Various functions and their relevance for important phenomena in life and diseases. Exp Cell Res 334: 16-25, 2015.

7. Camlin NJ, McLaughlin EA and Holt JE: Motoring through: The role of kinesin superfamily proteins in female meiosis. Hum Reprod Update 23: 409-420, 2017.

8. Samejima K, Samejima I, Vagnarelli P, Ogawa H, Vargiu G, Kelly DA, de Lima Alves F, Kerr A, Green LC, Hudson DF, et al: Mitotic chromosomes are compacted laterally by KIF4 and condensin and axially by topoisomerase II $\alpha$. J Cell Biol 199: $755-770,2012$

9. Mazumdar M, Sung MH and Misteli T: Chromatin maintenance by a molecular motor protein. Nucleus 2: 591-600, 2011.
10. Xue D, Cheng P, Han M, Liu X, Xue L, Ye C, Wang K and Huang J: An integrated bioinformatical analysis to evaluate the role of KIF4A as a prognostic biomarker for breast cancer. OncoTargets Ther 11: 4755-4768, 2018.

11. Taniwaki M, Takano A, Ishikawa N, Yasui W, Inai $K$, Nishimura H, Tsuchiya E, Kohno N, Nakamura Y and Daigo Y: Activation of KIF4A as a prognostic biomarker and therapeutic target for lung cancer. Clin Cancer Res 13: 6624-6631, 2007.

12. Matsumoto Y, Saito M, Saito K, Kanke Y, Watanabe Y, Onozawa H, Hayase S, Sakamoto W, Ishigame T, Momma T, et al: Enhanced expression of KIF4A in colorectal cancer is associated with lymph node metastasis. Oncol Lett 15: 2188-2194, 2018.

13. Hu G, Yan Z, Zhang C, Cheng M, Yan Y, Wang Y, Deng L, Lu Q and Luo S: FOXM1 promotes hepatocellular carcinoma progression by regulating KIF4A expression. J Exp Clin Cancer Res 38: 188, 2019.

14. Namløs HM, Meza-Zepeda LA, Barøy T, Østensen IH, Kresse SH, Kuijjer ML, Serra M, Bürger H, Cleton-Jansen AM and Myklebost O: Modulation of the osteosarcoma expression phenotype by microRNAs. PLoS One 7: e48086, 2012.

15. Cates JM: Simple staging system for osteosarcoma performs equivalently to the AJCC and MSTS systems. Journal of orthopaedic research : official publication of the Orthopaedic Research Society 36: 2802-2808, 2018.

16. Schmittgen TD and Livak KJ: Analyzing real-time PCR data by the comparative C(T) method. Nat Protoc 3: 1101-1108, 2008.

17. Mu J, Fan L, Liu D and Zhu D: Overexpression of shugoshin1 predicts a poor prognosis for prostate cancer and promotes metastasis by affecting epithelial-mesenchymal transition. OncoTargets Ther 12: 1111-1118, 2019.

18. Narayan G, Bourdon V, Chaganti S, Arias-Pulido H, Nandula SV, Rao PH, Gissmann L, Dürst M, Schneider A, Pothuri B, et al: Gene dosage alterations revealed by cDNA microarray analysis in cervical cancer: Identification of candidate amplified and overexpressed genes. Genes Chromosomes Cancer 46: 373-384, 2007.

19. Gao J, Sai N, Wang C, Sheng X, Shao Q, Zhou C, Shi Y, Sun S, Qu X and Zhu C: Overexpression of chromokinesin KIF4 inhibits proliferation of human gastric carcinoma cells both in vitro and in vivo. Tumour Biol 32: 53-61, 2011.

20. Minakawa Y, Kasamatsu A, Koike H, Higo M, Nakashima D, Kouzu Y, Sakamoto Y, Ogawara K, Shiiba M, Tanzawa H, et al: Kinesin family member $4 \mathrm{~A}$ : A potential predictor for progression of human oral cancer. PLoS One 8: e85951, 2013.

21. Zou JX, Duan Z, Wang J, Sokolov A, Xu J, Chen CZ, Li JJ and Chen HW: Kinesin family deregulation coordinated by bromodomain protein ANCCA and histone methyltransferase MLL for breast cancer cell growth, survival, and tamoxifen resistance. Mol Cancer Res 12: 539-549, 2014.

22. Pan J, Lei $X$ and Mao X: Identification of KIF4A as a pan-cancer diagnostic and prognostic biomarker via bioinformatics analysis and validation in osteosarcoma cell lines. PeerJ 9: e11455, 2021.

23. Mazumdar M, Sundareshan S and Misteli T: Human chromokinesin KIF4A functions in chromosome condensation and segregation. J Cell Biol 166: 613-620, 2004.

24. Liu G, Lu Y, Li L, Jiang T, Chu S, Hou P, Bai J and Chen M: The kinesin motor protein KIF4A as a potential therapeutic target in renal cell carcinoma. Invest New Drugs 38: 1730-1742, 2020.

This work is licensed under a Creative Commons Attribution-NonCommercial-NoDerivatives 4.0 International (CC BY-NC-ND 4.0) License. 